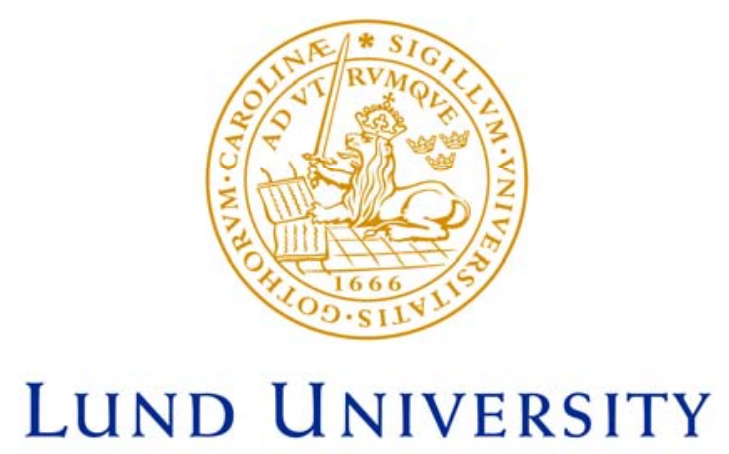

Faculty of Medicine

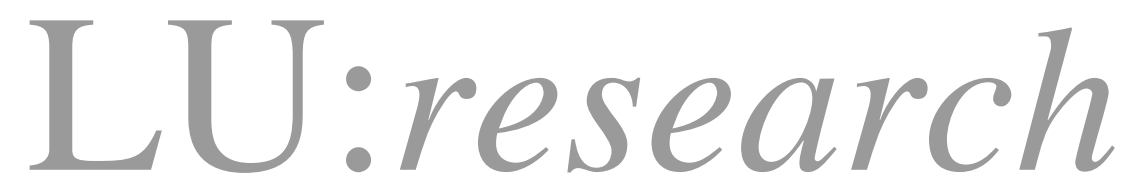

Institutional Repository of Lund University

This is an author produced version of a paper published in Biochemical and biophysical research communications. This paper has been peer-reviewed but does not include the final publisher proof-corrections or journal pagination.

Citation for the published paper:

Oknianska, Alina and Zmuda-Trzebiatowska, Emilia and Manganiello, Vincent and Degerman, Eva.

"Long-term regulation of cyclic nucleotide phosphodiesterase type 3B and 4 in 3T3-L1 adipocytes" Biochem Biophys Res Commun, 2007, Vol: 353, Issue: 4, pp. 1080-5.

http://dx.doi.org/10.1016/j.bbrc.2006.12.141

Access to the published version may require journal subscription.

Published with permission from: Elsevier 


\title{
Long-term regulation of cyclic nucleotide phosphodiesterase type 3B and 4 in 3T3-L1 adipocytes
}

\author{
Alina Oknianska*, Emilia Zmuda-Trzebiatowska*, Vincent Manganiello**, Eva \\ Degerman* \\ *Biomedical Center, C11, Department of Experimental Medical Science, 22184 Lund, \\ Sweden **Pulmonary Critical Care Medicine Branch, NHLB, NIH, Bethesda, Maryland, \\ 20892, USA
}

Emilia Zmuda-Trzebiatowska

Biomedical Center, $\mathrm{C} 11$

Department of Experimental Medical Science

22184 Lund, Sweden

Tel: +46462229552

Fax: +46462224022

e-mail: emilia.zmuda-trzebiatowska@med.lu.se

Keywords

phosphodiesterase 3B, phosphodiesterase 4, adipocyte, insulin, isoproterenol 


\begin{abstract}
Phosphodiesterase 3B (PDE3B), known to play an important role in acute insulin and cAMP-mediated regulation of lipid metabolism, and PDE4 are the main PDE types expressed in adipocytes. Here we show that members of all PDE4 isoforms are expressed in 3T3-L1 and primary mouse adipocytes. Long-term treatment of 3T3-L1 adipocytes with insulin induced up-regulation of PDE3B and PDE4D in a phosphatidylinositol 3kinase-dependent manner whereas long-term treatment with $\beta$-adrenergic agonists induced down-regulation of PDE3B and up-regulation of PDE4D. Thus, PDE3B and PDE4D can be added to the list of genes regulated by insulin and cAMP-increasing hormones. Altered expression of PDE3B and PDE4D in response to long-term treatment with insulin and catecholamines may contribute to altered regulation of metabolism in diabetes.
\end{abstract}

\title{
Introduction
}

Cyclic nucleotide phosphodiesterases (PDEs) regulate crucial cellular signalling pathways through hydrolysis of key second messengers, cAMP and cGMP. Eleven PDE families (PDE1-11) have been identified based on their substrate affinities, biochemical and physical properties, mechanisms whereby they are regulated and different sensitivities to inhibitors [1, 2]. Adipocytes express membrane-bound PDE3B and membrane bound as well as cytosolic PDE4s [3]. Insulin and cAMP-mediated activation of PDE3B has been shown to play an important role in the regulation of lipolysis, lipogenesis and glucose uptake in adipocytes [4-6]. Mechanisms for short-term regulation 
of PDE3B by insulin have been extensively studied and involve phosphatidylinositol 3kinase (PI3K)-dependent phosphorylation and activation of the enzyme catalyzed by protein kinase B [7]. Mechanisms for short-term regulation of PDE3B by cAMP may involve protein kinase A, Epacs (Exchange proteins directly activated by cAMP) and PKB [4-6].

Less has been elucidated regarding long-term regulation of adipocyte PDE3B. Two promoter regions of PDE3B have recently been characterized and cAMP response element (CRE) binding protein (CREB) appeared to play an important role in mediating up-regulation of PDE3B during differentiation of pre-adipocytes into mature adipocytes [8]. Further, PDE3B has been shown to be down-regulated in adipocytes from obese, insulin resistant $\mathrm{cp}$ rats [9], diabetic, obese KKAy mice [10] and obese $\mathrm{db} / \mathrm{db}$ mice [11]. Interestingly, peroxisome proliferator-activated receptor $\gamma$ (PPAR $\gamma$ ) agonists, used as insulin-sensitizing drugs, were able to restore PDE3B levels [10-12]. With regard to possible mediators of PDE3B down-regulation, it has been demonstrated that prolonged treatment of 3T3-L1 adipocytes with TNF- $\alpha$, a cytokine which is up-regulated in obese/diabetic conditions, and ceramide, a suggested mediator of TNF- $\alpha$ action [13], caused down-regulation of PDE3B $[14,15]$. These effects were reversed in the presence of troglitazone (PPAR $\gamma$-agonist) [15]. Down-regulation of PDE3B in 3T3-L1 adipocytes has also been observed after long-term treatment with dbcAMP, dexamethazone and triiodothyronin [16].

Very little is known about roles and acute as well as long-term regulation of different PDE4 isoforms (PDE4A, B, C, D, long and short variants, respectively [17]) in 
adipocytes. In other cell types, regulation of PDE4 has been shown to occur at the level of phosphorylation, protein interactions and proteolysis [18].

In this study, we have investigated long-term regulation of adipocyte PDEs under diabetic conditions, mimicked by prolonged exposition of 3T3-L1 adipocytes to high levels of glucose, insulin and $\beta$-adrenergic agonists.

\section{Materials and methods}

Materials. 3T3-L1 cells were from American Type Culture Collection (Manassas, VA). Dulbecco's modified Eagle's medium (DMEM), Penicillin/ Streptomycin (P/S) and foetal calf serum (FCS) were from Gibco BRL. The $\beta 3$-adrenergic receptor agonist CL 316243 and 3-isobutyl-1-methylxantine (IBMX) were from Sigma. The PDE4 inhibitor, RO 20-1724 was from Biomol and the PDE3 inhibitor, OPC 3911, was a kind gift from Otsuka Pharmaceuticals, Tokyo, Japan.

Cell culture and preparation of adipocyte homogenates. 3T3-L1 fibroblasts (80,000-100,000 per $100 \mathrm{~mm}$ plate) were cultured in $10 \mathrm{ml}$ of DMEM containing $10 \%$ FCS and $1 \% \mathrm{P} / \mathrm{S}$ at $37{ }^{\circ} \mathrm{C}$ under humidified atmosphere (air/ $\left.\mathrm{CO}_{2}-19: 1\right)$. Confluent cells were differentiated for 2 days in DMEM supplemented with $0.5 \mathrm{mM}$ IBMX, $10 \mu \mathrm{g} / \mathrm{ml}$ insulin and $1 \mu \mathrm{M}$ dexamethasone. Experiments were performed with 3T3-L1 adipocytes (80-95\% differentiated) 8-14 days after the initiation of differentiation. Adipocytes were washed and homogenized at $4^{\circ} \mathrm{C}$ in $300 \mu \mathrm{l}$ of homogenization buffer $(25 \mathrm{mM}$ Tris/ $\mathrm{HCl}$, 
pH 7.5, $250 \mathrm{mM}$ sucrose, $5 \mathrm{mM} \mathrm{MgCl}_{2}, 0.2 \mathrm{mM}$ EGTA, $20 \mathrm{mM} \beta$-glycerophosphate, 20 $\mathrm{mM}$ phenylphosphate, $1 \mu \mathrm{g} / \mathrm{ml}$ of pepstatin $\mathrm{A}, 10 \mu \mathrm{g} / \mathrm{ml}$ of antipain and leupeptin). Homogenates were centrifuged (33000xg) for $45 \mathrm{~min}$ at $4{ }^{\circ} \mathrm{C}$ and the fractions (cytosols and membranes rehomogenized in $300 \mu$ of the homogenization buffer) were collected.

SDS-page and western blot analysis. Samples containing twenty (membrane fraction) and thirty (cytosolic fraction) micrograms of protein measured according to [19] were subjected to polyacrylamide gel electrophoresis (PAGE) (10\% acrylamide) and western blot analysis. Polyvinylidene difluoride membranes (Millipore) were blocked for 30 min with $5 \%$ milk in TBS-T buffer (50 mM Tris $\mathrm{pH} 7.5,150 \mathrm{mM} \mathrm{NaCl}, 0.1 \%$ (w/v) Tween-20), and incubated overnight with either a 1: 1000 dilution of anti-PDE3B antibodies (raised in rabbits as described in [20]), or a 1: 5000 dilution of anti-PDE4A, B, C and D antibodies (a kind gift from Professor Miles Houslay). Washed membranes were incubated for $1 \mathrm{~h}$ with 1:5000 dilution of anti-rabbit antibodies linked with Horseradish peroxidase (Amersham), washed again and developed with the 1:1 solution of Peroxide and Luminol (Pierce). The chemoluminescent signal was analyzed and quantified with use of the Fuji LAS 1000 Plus system (Fuji Photo Film, Tokyo, Japan).

PDE activity measurements. PDE3 activity was measured as described previously [21] in the presence or absence of $3 \mu \mathrm{M}$ OPC 3911 (PDE3 inhibitor) or $10 \mu \mathrm{M}$ RO 201724 (PDE4 inhibitor). 
Statistics. Data are expressed as mean values +/- SEM. of the indicated number of experiments. Statistical significance (here as $\mathrm{p}<0.05$ ) was evaluated with use of unpaired Student's $t$ test and showed as stars in the figures.

\section{Results}

Long-term treatment of 3T3-L1 adipocytes with insulin results in up-regulation of $P D E 3 B$ and PDE4D

It has previously been shown that adipocytes express a $135 \mathrm{kDa}$ membrane-bound PDE3B [22]. PDE4 is also known to be expressed in this cell type [3], however very little is known with regard to the expression pattern of adipocyte PDE4 isoforms. As shown in Fig. 1A, the majority of PDE4 activity was detected in the cytosolic fractions of 3T3-L1 adipocytes. Furthermore, long forms of all PDE4 isoforms (PDE4A-D) were detected in 3T3-L1 as well as in primary mouse adipocytes, mainly in the cytosolic fractions (Fig. 1B).

As shown in Fig. 2A, long-term treatment of 3T3-L1 adipocytes with insulin resulted in increased PDE3B activity in the membrane fraction (1.3-fold and 2.2-fold, after 24 and 46 hours respectively). Up-regulation of PDE3B activity was associated with increased amount of PDE3B protein (Fig. 2B). PDE3B protein was up-regulated 2-fold after 24 hours and 4-fold after 46 hours of exposition to insulin.

Prolonged treatment of 3T3-L1 adipocytes with insulin caused an increase in PDE4 activity in cytosols from 3T3-L1 adipocytes (2-fold after 24 hours and 1.5-fold after 46 
hours) (Fig. 2C). This increase in activity was associated with increased amount of PDE4D protein (Fig. 2D). PDE4D protein was up-regulated almost 5-fold after 24 hours and 2-fold after 46 hours of insulin treatment. No alterations in PDE4 A, B or C expression was detected (data not shown).

Inhibition of PI3K abolishes insulin-induced up-regulation of PDE3B and PDE4D

As shown in Fig. 3A, incubation of 3T3-L1 adipocytes with insulin in the presence of the PI3K inhibitor, LY 294002, for 46 hours, resulted in a 60\% decrease in insulin-induced PDE3B activity. Furthermore, exposition of the cells to LY 294002 alone resulted in a slightly decreased basal PDE3 activity and a lowering of the PDE3B expression by $35 \%$ (Fig. 3B).

Incubation of 3T3-L1 adipocytes with insulin in the presence of LY 294002 for 24 hours, entirely prevented insulin-induced increase in PDE4 activity and reduced protein expression by $60 \%$ (Fig. 3C, D). The basal PDE4 activity and protein expression was lowered by 10 and $6 \%$, respectively in comparison to untreated cells (Fig 3D).

Long-term treatment of 3T3-L1 adipocytes with $\beta$-adrenergic receptor agonists induces down-regulation of $P D E 3 B$ and up-regulation of $P D E 4 D$

As shown in Fig. 4A, 46 hour-treatment of 3T3-L1 adipocytes with isoproterenol, a general $\beta$-adrenergic receptor agonist or CL316243, a $\beta 3$ AR-specific agonist, caused a decrease in PDE3B activity which was associated with reduced PDE3B protein 
expression as compared to the control cells (Fig. 4B). Thus, PDE3 activity was reduced by $30 \%$ after a 2 hour-treatment with isoproterenol and this effect was maintained for at least 46 hours (Fig. 4B). Reduced PDE3B protein expression was observed after 2 and 46 hours of isoproterenol treatment (40\% reduction after $46 \mathrm{~h})$. Furthermore, stimulation of 3T3-L1 adipocytes with CL 316243 caused a 40\% decrease in PDE3B activity after a 2 hour-treatment which was also associated with decreased PDE3B protein expression. Long-term $\beta$-adrenergic receptor stimulation had the opposite, stimulatory effects on PDE4 activity and PDE4D protein expression. After 3 hours, isoproterenol and CL 316243 stimulation increased PDE4 activity 1.5-fold and 1.6-fold whereas after 24 hours PDE4 activity was increased 2-fold and 1.4-fold, respectively (Fig. 4C). Increased PDE4 activity was associated with increased expression of PDE4D protein, 2-fold after 24 hours, as shown in Fig. 4D. No changes were observed with regard to the expression of PDE4A, B, C (data not shown).

\section{Discussion}

New findings regarding long-term regulation of PDE3 and PDE4 in 3T3-L1 adipocytes treated with insulin and $\beta$-adrenergic agonists are presented in this study. Thus, insulin induced up-regulation of PDE3B as well as PDE4D, whereas $\beta$-adrenergic agonists induced down-regulation of PDE3B and up-regulation of PDE4D. As for other PDE4 isoforms (PDE4A, B, C) also expressed in adipocytes, no hormone-induced alterations in gene expression were observed. Hence, PDE3B and PDE4D can be added to the list of genes regulated by both insulin and cAMP-increasing hormones. Obviously, long-term 
treatment of cells with insulin and catecholamines alters the expression of many genes regulating energy homeostasis and metabolism, and the exact contribution of altered expression of PDE3B and PDE4D in this context is not known. However, it is known that PDE3B knockout mice show a number of derangements in energy homeostasis, including insulin resistance [7]. As for PDE4, down-regulation of PDE4A appeared to contribute to increased catecholamine sensitivity in adipocytes and to the lean phenotype of mice overexpressing the FOXC2 transcription factor in adipocytes [23]. Thus, altered expression of PDE3B and PDE4 may very well significantly contribute to altered regulation of metabolism.

Insulin-induced up-regulation of PDE3B was found to be PI3K-dependent. Although insulin response elements (IRE) have recently been identified in the PDE3B promoter region [8], it is not yet known which transcription factors/ co-activators are involved in mediating the effect of insulin on PDE3B gene expression. A few transcription factors phosphorylated in response to insulin have been identified but none have been unequivocally proven to mediate the effect of insulin on gene transcription. Thus, it has recently been shown that insulin stimulates Ser 133 phosphorylation of CREB and thereby enhances CREB transcriptional activity in HepG2 (human hepatoma) cells, mouse 3T3 L1 fibroblasts and adipocytes [24]. This is interesting in the context of this work, since it has recently been demonstrated that CREB is involved in mediating PDE3B expression during differentiation of 3T3-L1 adipocytes induced by insulin, dbcAMP and dexamethasone [8].

Whereas in this study long-term treatment with high insulin induced up-regulation of PDE3B activity and protein, PDE3B protein has previously been shown to be down- 
regulated in adipose tissue/ adipocytes of several models of diabetes and obesity [9-11]. Therefore, based on the findings from this study, one can conclude that hyperinsulinemia per se does not contribute to the down-regulation of PDE3B observed in vivo. The situation is obviously very complex in the in vivo models where adipocytes are exposed to and differentially affected by an altered environment characterized by a combination of hyperinsulinemia, hyperglycemia, increased fatty acids, increased levels of catecholamines and altered levels of circulating cytokines and other factors [25, 26]. With regard to some of theses conditions one should note that 3T3-L1 adipocytes were cultured in high glucose $(25 \mathrm{mM})$; lowering glucose to $5 \mathrm{mM}$ was associated with a reduction in the effect of insulin on PDE3B gene expression (data not shown), indicating a positive cross-talk between glucose and insulin in increasing PDE3B gene expression. Increasing the level of oleic acid up to $2 \mathrm{mM}$ for more than 48 hours did not alter PDE3B gene expression in 3T3-L1 adipocytes (data not shown). However, as discussed below, $\beta$ adrenergic agonists indeed reduced PDE3B gene expression, in agreement with a previous study showing that dbcAMP reduced PDE3B gene expression in 3T3-L1 adipocytes [14]. Therefore, increased levels of catecholamines in vivo possibly contribute to the down-regulation of PDE3B observed in animal models of diabetes and obesity. Also TNF $\alpha$ which has been shown to reduce PDE3B gene expression in mouse 3T3-L1 cells and in human adipocytes after differentiation from preadipocytes [14] could contribute to the down-regulation of PDE3B observed in the in vivo models.

Mechanisms whereby $\beta$-adrenergic receptor agonists cause down-regulation of PDE3B are not known. CREB, a transcriptional factor mediating effects of cAMP and other signalling molecules, has previously been shown to play a critical role in inducing 
PDE3B gene expression during differentiation of 3T3-L1 adipocytes in a PKA-dependent manner [8]. One could speculate that sustained down-regulation of PDE3B might be the result of CREB-mediated up-regulation of ICER proteins (inducible cAMP early repressors; members of the CRE modulator family) as it was demonstrated to be the case for PDE3A in cardiomyocytes [27]. ICER proteins function as endogenous inhibitors of gene transcription (including their own) mediated by CREB, by competing with CREB in binding CRE sequences [27]. In the above mentioned study it has been shown that PDE3A expression and activity is significantly down-regulated in human failing hearts and that down-regulation of PDE3A leads to apoptosis due to the induction of ICER. Thus, an autoregulatory positive feedback mechanism of cardiomyocyte apoptosis has been identified: ICER represses PDE3A gene expression and PDE3A reduction in turn enhances ICER expression due to PKA activation [27]. Whether there is an interplay between PDE3B-CREB-ICER in adipocytes is, however, not yet known.

As is the case for PDE3B, PDE4 activity and protein (PDE4D specifically) also was increased in a PI3K-dependent manner by long-term treatment with insulin. Inhibition of PDE4 but not PDE3 has been shown to be important during the differentiation of preadipocytes to mature adipocytes and PDE4 expression appeared to decrease during the differentiation process [28]. In general, very little is known about the role of PDE4 in adipocytes and particularly little is known about insulin in relation to PDE4s. It has been established that ERK, a kinase belonging to the MAP kinase pathway, which is activated by insulin in adipocytes, can influence PDE4 activity [17]. Thus, ERK acutely induces phosphorylation of several PDE4 isoforms. Phosphorylation of short versions of PDE4B and $\mathrm{D}$ results in activation of enzyme activity whereas phosphorylation of long versions 
of PDE4B, C and D results in their inhibition [17]. The inhibitory effect of ERK is transient, as the increase in cAMP caused by PDE4 inhibition activates PKA, which in turn phosphorylates and activates PDE4s [17], an example of hormone induced crosstalk between different PDEs which most likely can also occur in our system between PDE3B and PDE4D.

The stimulatory effects of $\beta$-adrenergic receptor agonists on PDE4D expression might be mediated by CREB since CRE elements have been identified in the PDE4D promoter [29]. In cells other than adipocytes it has also been shown that prolonged exposition to cAMP-increasing agents results in PDE4D up-regulation. Thus, PDE4D appeared to be induced after long-term treatment of thyroid cells and Sertoli cells with thyroid stimulating hormone and folliculate stimulating hormone respectively. Similar results were obtained for Jurkat $\mathrm{T}$ cells and rat skeletal myoblasts. cAMP effects on PDE4D upregulation could also be mimicked by forskolin [reviewed in 18]. Recently, in vascular smooth muscle cells (VSMC), phenotype-selective expression of PDE4D isoforms has been demonstrated. Thus, cAMP elevating agents were found to up-regulate the expression of PDE4D3 in contractile VSMC, whereas the short PDE4D1 and PDE4D2 variants were selectively induced in synthetic VSMC. These expression patterns appeared to be dependent on histone acetylation status of their promoters [30]. A number of studies on genetically modified animals and on animal models for obesity/ diabetes show that both PDE3B and PDE4 play important roles in the regulation of energy homeostasis. Thus, altered expression of PDE3B as well as PDE4D in response to long-term treatment with insulin and catecholamines may contribute to altered regulation of metabolism in diabetic states. 


\section{Figure legend}

Fig. 1. Activity and expression of PDE4 isoforms in primary and 3T3-L1 adipocytes. PDE 4 activity was measured in membrane and cytosolic fractions of adipocytes (A) and expressed as means +/- SEM. Western blots of PDE4 isoforms in primary and 3T3-L1 adipocytes are shown (B).

Fig. 2. PDE3B and PDE4D are up-regulated in response to insulin in 3T3-L1 adipocytes. 3T3-L1 adipocytes were treated with $100 \mathrm{nM}$ insulin for 24 or $46 \mathrm{~h}$. PDE3 activity in membrane fractions (20 $\mu \mathrm{g}$ protein) (A) and PDE4 activity in cytosol (30 $\mu \mathrm{g}$ protein) $(\mathrm{C})$ were measured and expressed as means \pm SEM, $\mathrm{n}=3-5 ; *$ significantly different from untreated cells $(\mathrm{Ctr} ; 100 \%=34 \mathrm{pmol} / \mathrm{min} / \mathrm{mg}$ for PDE3 and $21 \mathrm{pmol} / \mathrm{min} / \mathrm{mg}$ for PDE4). Western blots of PDE3B and PDE4D are shown (B, D), representative of 4 and 3 experiments, respectively.

Fig. 3. Insulin-induced up-regulation of PDE3B and PDE4D is decreased in the presence of LY 294002 in 3T3-L1 adipocytes. 3T3-L1 adipocytes were treated with100 nM insulin or10 $\mu$ M LY 294002 alone or with insulin for 46h. PDE3 activity in membrane fractions (20 $\mu \mathrm{g}$ protein) $(\mathrm{A})$ and PDE4 activity in cytosol (30 $\mu \mathrm{g}$ protein) $(\mathrm{C})$ were measured and expressed as means $\pm \mathrm{SEM}, \mathrm{n}=3-5$; $\mathrm{Ctr}(100 \%)=32 \mathrm{pmol} / \mathrm{min} / \mathrm{mg}$ for PDE3 and 21 $\mathrm{pmol} / \mathrm{min} / \mathrm{mg}$ for PDE4; * significantly different from insulin-treated cells (ins). Western blots of PDE3B and PDE4D are shown (B, D), representative of 3 experiments. 
Fig. 4. PDE3B is down-regulated whereas PDE4D is up-regulated in response to cAMPincreasing hormones in 3T3-L1 adipocytes. 3T3-L1 adipocytes were treated for 2, 3 or 46h with $100 \mathrm{nM}$ isoproterenol or $100 \mathrm{nM}$ CL 316243 as well as with $100 \mathrm{nM}$ insulin as control. PDE3 activity in membrane fractions (20 $\mu$ g protein) (A) and PDE4 activity in cytosol (30 $\mu$ g protein) $(\mathrm{C})$ were measured and expressed as means $\pm \mathrm{SEM}, \mathrm{n}=3-7$; * significantly different from untreated cells $(\mathrm{Ctr}(100 \%)=46 \mathrm{pmol} / \mathrm{min} / \mathrm{mg}$ for PDE3 and $20 \mathrm{pmol} / \mathrm{min} / \mathrm{mg}$ for PDE4). Western blots of PDE3B and PDE4D are shown (B, D), representative of 3 experiments.

\section{References}

[1] S.H. Soderling, J.A. Beavo, Regulation of cAMP and cGMP signaling: new phosphodiesterases and new functions, Curr. Opin. Cell. Biol. 12 (2000) 174-179.

[2] S.H. Francis, I.V. Turko, J.D. Corbin, Cyclic nucleotide phosphodiesterases: relating structure and function, Prog. Nucleic Acid Res. Mol. Biol. 65 (2001) 1-52.

[3] C. Schmitz-Peiffer, M.L. Reeves, R.M. Denton, Characterization of the cyclic nucleotide phosphodiesterase isoenzymes present in rat epididymal fat cells, Cell. Signal. 4 (1992) 37-49.

[4] E. Zmuda-Trzebiatowska, A. Oknianska, V. Manganiello, E. Degerman, Role of PDE3B in insulin-induced glucose uptake, GLUT-4 translocation and lipogenesis in primary rat adipocytes, Cell. Signal. 18 (2006) 382-390. 
[5] Y. Shakur, L.S. Holst, T.R. Landstrom, M. Movsesian, E. Degerman, V. Manganiello, Regulation and function of the cyclic nucleotide phosphodiesterase (PDE3) gene family, Prog Nucleic Acid Res. Mol. Biol. 66 (2001) 241-277.

[6] Y. Choi, S. Park, S. Hockman, E Zmuda-Trzebiatowska., F. Svennelid, M. Haluzik O., Gavrilova L., Peppin, M. Napolitano, M. Taira, F. Ahmad L.S., Holst, E. Degerman, V. Manganiello, Alterations in regulation of energy homeostasis in cyclic nucleotide phosphodiesterase 3B-null mice, J. Clin. Invest., in press

[7] E. Degerman, C.J. Smith, H. Tornqvist, V. Vasta, P. Belfrage, V.C. Manganiello, Evidence that insulin and isoprenaline activate the cGMP-inhibited low-Km cAMP phosphodiesterase in rat fat cells by phosphorylation, Proc. Natl. Acad. Sci. U S A 87 (1990) 533-537.

[8] H. Liu, J.R. Tang, Y.H. Choi, M. Napolitano, S. Hockman, M. Taira, E. Degerman, V.C. Manganiello, Importance of cAMP-response element-binding protein in regulation of expression of the murine cyclic nucleotide phosphodiesterase 3B (Pde3b) gene in differentiating 3T3-L1 preadipocytes, J. Biol. Chem. 281 (2006) 21096-21113.

[9] T. Nagaoka, T. Shirakawa, T.W. Balon, J.C. Russell, Y. Fujita-Yamaguchi, Cyclic nucleotide phosphodiesterase 3 expression in vivo: evidence for tissue-specific expression of phosphodiesterase $3 \mathrm{~A}$ or $3 \mathrm{~B}$ mRNA and activity in the aorta and adipose tissue of atherosclerosis-prone insulin-resistant rats, Diabetes 47 (1998) 1135-1144.

[10] Y. Tang, H. Osawa, H. Onuma, M. Hasegawa, T. Nishimiya, M. Ochi, H.Makino, Adipocyte-specific reduction of phosphodiesterase 3B gene expression and its restoration by JTT-501 in the obese, diabetic KKAy mouse, Eur. J. Endocrinol. 145 (2001) 93-99. 
[11] Y. Tang, H. Osawa, H. Onuma, T. Nishimiya, M. Ochi, A.Sugita, H. Makino, Phosphodiesterase 3B gene expression is enhanced in the liver but reduced in the adipose tissue of obese insulin resistant db/db mouse, Diabetes Res. Clin. Pract. 54 (2001) 145155.

[12] P. Engfeldt, P. Arner, J. Bolinder, J. Ostman, Phosphodiesterase activity in human subcutaneous adipose tissue in insulin- and noninsulin-dependent diabetes mellitus, J. Clin. Endocrinol. Metab. 55 (1982) 983-988.

[13] D.N. Brindley, C.N. Wang, J. Mei, J. Xu, A.N. Hanna, Tumor necrosis factor-alpha and ceramides in insulin resistance, Lipids 34 (1999) Suppl.S 85-88.

[14] T. Rahn Landstrom, J. Mei, M. Karlsson, V. Manganiello, E. Degerman, Downregulation of cyclic-nucleotide phosphodiesterase 3B in 3T3-L1 adipocytes induced by tumour necrosis factor alpha and cAMP, Biochem. J. 346 (2000) 337-343.

[15] J. Mei, L.S. Holst, T.R. Landstrom, C. Holm, D. Brindley, V. Manganiello, E. Degerman, $\mathrm{C}(2)$-ceramide influences the expression and insulin-mediated regulation of cyclic nucleotide phosphodiesterase 3B and lipolysis in 3T3-L1 adipocytes, Diabetes 51 (2002) 631-637.

[16] M.L. Elks, V.C. and Manganiello, Effects of thyroid hormone on regulation of lipolysis and adenosine 3',5'-monophosphate metabolism in 3T3-L1 adipocytes, Endocrinology 117 (1985) 947-953.

[17] M.D. Houslay, PDE4 cAMP-specific phosphodiesterases, Prog. Nucleic Acid Res. Mol. Biol. 69 (2001) 249-315. 
[18] C. Lugnier, Cyclic nucleotide phosphodiesterase (PDE) superfamily: a new target for the development of specific therapeutic agents, Pharmacol. Ther. 109 (2006) 366-398. [19] M.M. Bradford, A rapid and sensitive method for the quantitation of microgram quantities of protein utilizing the principle of protein-dye binding, Anal. Biochem. 72 (1976) 248-254.

[20] M. Taira, S.C. Hockman, J.C. Calvo, P. Belfrage, V.C. Manganiello, Molecular cloning of the rat adipocyte hormone-sensitive cyclic GMP-inhibited cyclic nucleotide phosphodiesterase. J. Biol. Chem. 268 (1993), 18573-18579.

[21] V.C. Manganiello, F. Murad, M. Vaughan, Effects of lipolytic and antilipolytic agents on cyclic 3',5'-adenosine monophosphate in fat cells. J. Biol. Chem. 246 (1971) $2195-2202$.

[22] E. Degerman, P. Belfrage, V.C. Manganiello, Structure, localization, and regulation of cGMP-inhibited phosphodiesterase (PDE3), J. Biol. Chem. 272 (1997) 6823-6826.

[23] L.M. Gronning, G.S. Baillie, A. Cederberg, M.J. Lynch, M.D. Houslay, S. Enerback, K. Tasken, Reduced PDE4 expression and activity contributes to enhanced catecholamine-induced cAMP accumulation in adipocytes from FOXC2 transgenic mice, FEBS Lett. 580 (2006) 4126-4130.

[24] D.J. Klemm, W.J. Roesler, T. Boras, L.A. Colton, K. Felder, J.E. Reusch, Insulin stimulates cAMP-response element binding protein activity in HepG2 and 3T3-L1 cell lines, J. Biol.Chem. 273 (1998) 917-923.

[25] L. Pirola, A.M. Johnston, E. Van Obberghen, Modulation of insulin action, Diabetologia 47 (2004) 170-184. 
[26] S.J. Bhathena, Relationship between fatty acids and the endocrine and neuroendocrine system, Nutr. Neurosci. 9 (2006) 1-10.

[27] B. Ding, J. Abe, H. Wei, H. Xu, W. Che, T. Aizawa, W. Liu, C.A. Molina, J. Sadoshima, B.C. Blaxall, B.C. Berk, C. Yan, A positive feedback loop of phosphodiesterase 3 (PDE3) and inducible cAMP early repressor (ICER) leads to cardiomyocyte apoptosis, Proc. Natl. Acad. Sci. U S A 102 (2005) 14771-14776.

[28] M.L. Elks, V.C. Manganiello, A role for soluble cAMP phosphodiesterases in differentiation of 3T3-L1 adipocytes, J. Cell. Physiol. 124 (1985) 191-198.

[29] I.R. Le Jeune, M. Shepherd, G. Van Heeke, M.D. Houslay, I.P. Hall, Cyclic AMPdependent transcriptional up-regulation of phosphodiesterase 4D5 in human airway smooth muscle cells. Identification and characterization of a novel PDE4D5 promoter, J. Biol. Chem. 277 (2002) 35980-35989.

[30] D.G. Tilley, D.H. Maurice, Vascular smooth muscle cell phenotype-dependent phosphodiesterase 4D short form expression: role of differential histone acetylation on cAMP-regulated function, Mol. Pharmacol. 68 (2005) 596-605. 


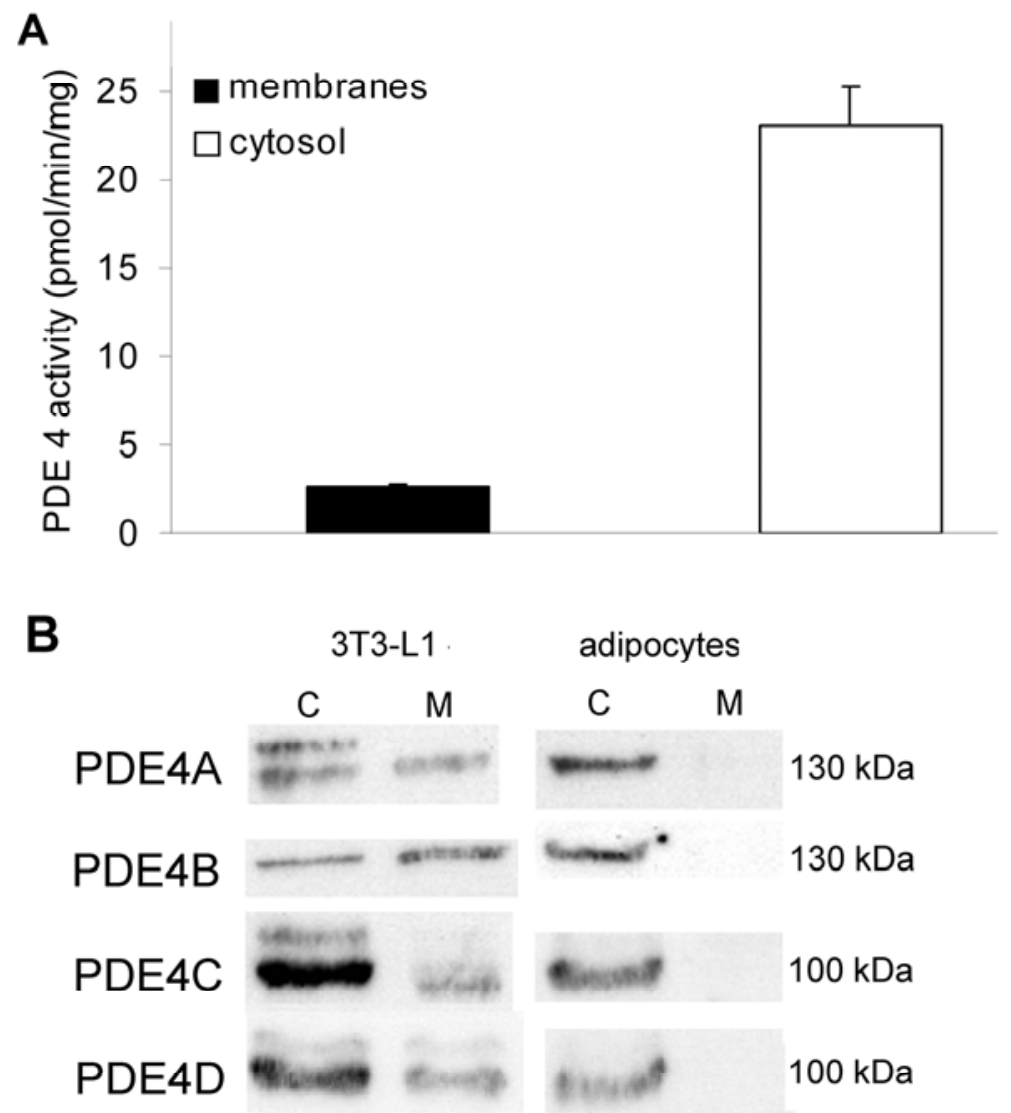

Fig. 1

A

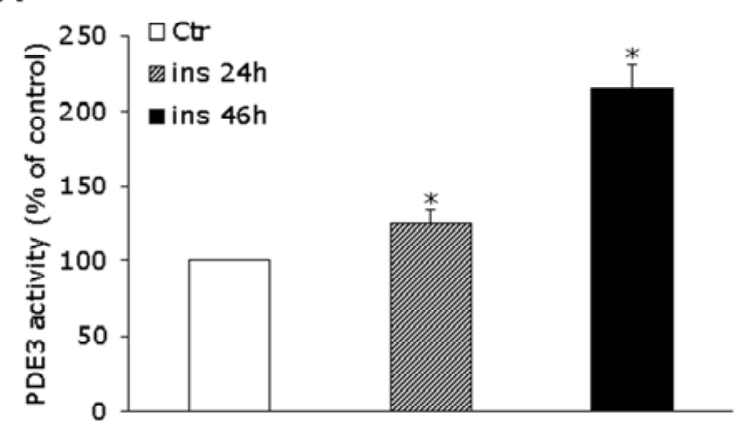

B

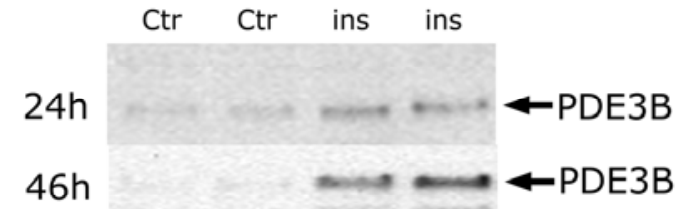

C

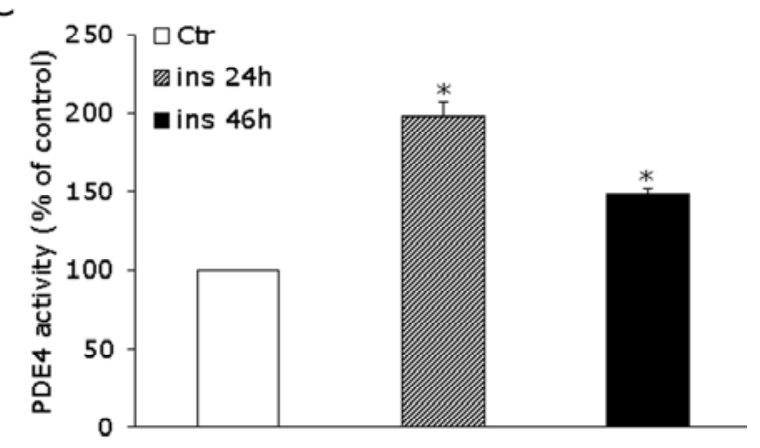

D

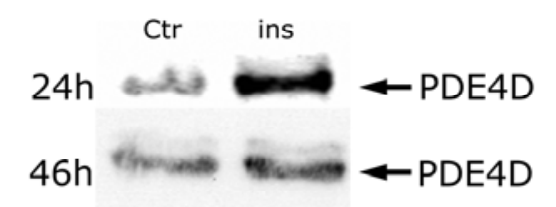

Fig. 2 


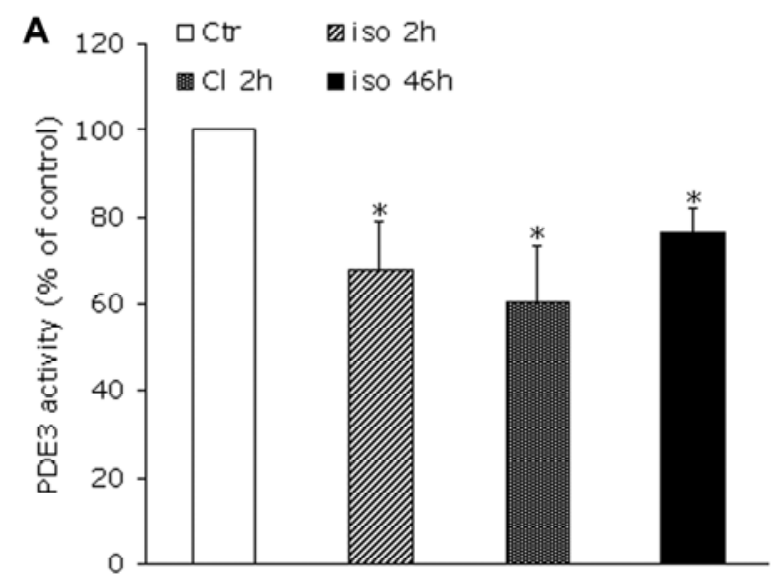

B

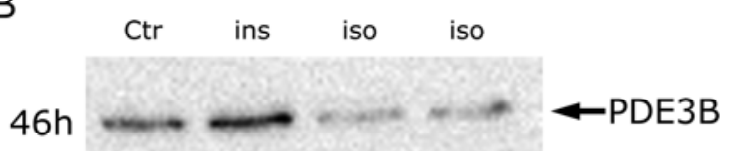

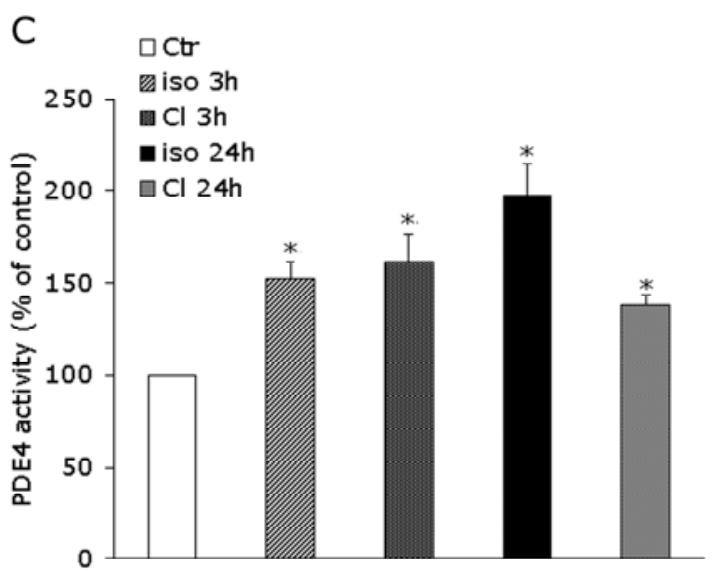

D

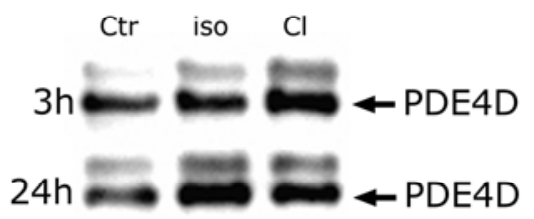

Fig. 3

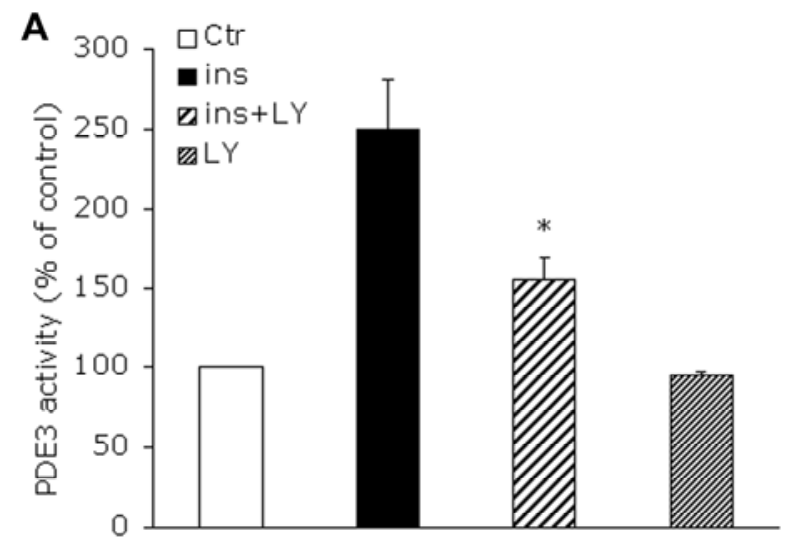

B

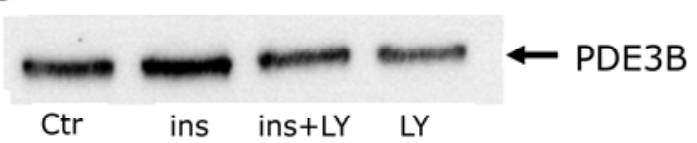

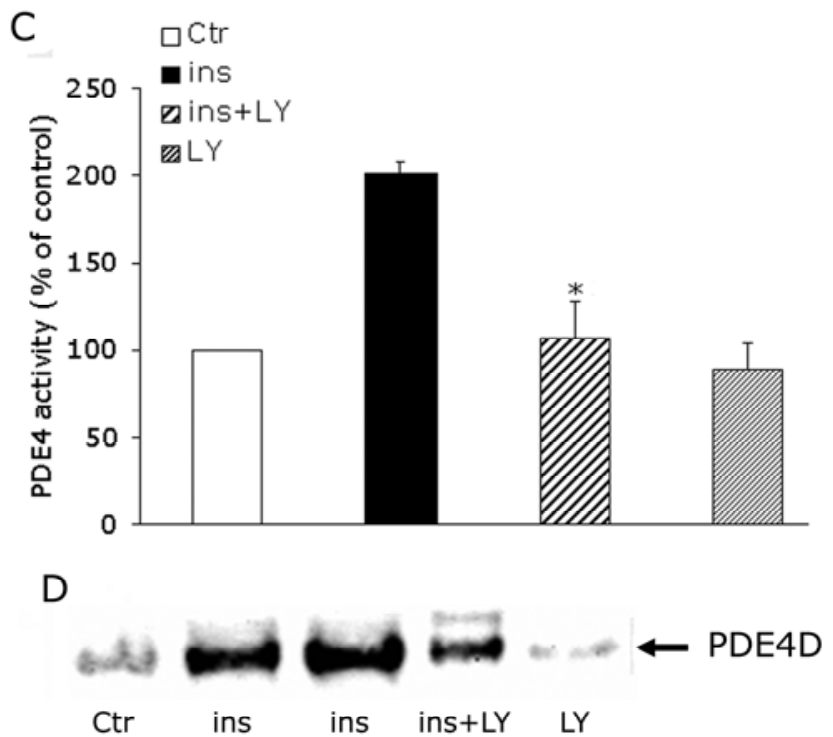

Fig. 4 